\title{
Venkatesh Vijayaraghavan Examines the Exciting Field of Computational Engineering
}

\section{Kaitlin Calva}

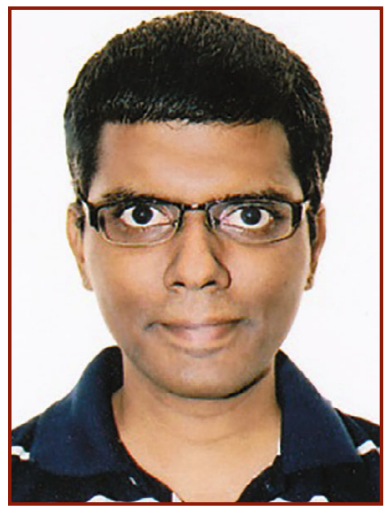

Venkatesh Vijayaraghavan

\section{young professional technical notes}

This occasional

feature highlights the scientific interests and professional accomplishments of a young TMS member who has contributed to the technical content of the current issue of JOM as an author, advisor, or guest editor. The development of this feature is a special project of the TMS Young Professionals Committee. For additional information contact Kaitlin Calva, JOM Magazine Managing Editor, at kcalva@tms.org.
"I have always been fascinated by computational techniques in engineering, regardless of domain, such as mechanical, structural, chemical, or energy engineering," said Venkatesh Vijayaraghavan, a faculty fellow at the University of New South Wales (UNSW). In his June 2020 paper, "Tensile Properties of Boron NitrideCarbon Nanosheet Reinforced Aluminum Nanocomposites using Molecular Dynamics Simulation," Vijayaraghavan explores mechanical properties of novel nanocomposites using computer simulation techniques.

"This paper highlights some of the critical aspects which should be considered to accurately characterize the mechanics of boron nitride nanocomposites using computational modelling," he said. "The modelling framework used in the article could be adopted by practicing materials engineers and scientists who can further improve upon it while working using more complex, multiscale models."

"For the case of mechanics of nanomaterials, it is indeed very difficult to conduct real-world experiments due to the scale of the material. As computational modelling has evolved as a powerful tool in estimating strength characteristics of novel nanostructures, it is also important to ensure the reliability which can promote its wide-scale applications," Vijayaraghavan explained. "Also, the other interesting fact about computational modelling is that it is possible to virtually design a completely new material and test its responses to a particular loading nature. With new advancements every day in computer infrastructures, this field has become even more exciting."

Looking at the myriad possibilities and challenges facing the rapidly changing field of computational modelling, Vijayaraghavan observed that "there is a significant opportunity for students and the academic community at large." However, he continued: "Equally challenging is the fact that it is also important to ensure how well the virtual engineering research can translate to a real-world scenario, since ultimately this is what it counts."

Noting the influence that mentoring has had on his career, Vijayaraghavan credited Wong Chee How, his Ph.D. advisor at Nanyang Technological University, with introducing him to the field of computational engineering. And his current mentor at UNSW, Liangchi Zhang, has been a great resource in "providing useful guidance whenever needed."

Vijayaraghavan also referenced other helpful resources for career guidance, such as his membership in TMS, where TMS journals have helped to keep him in touch with recent advancements in the field. He also thanked Nikhil Gupta, New York University, and Robert Maass, University of Illinois at Urbana-Champaign, for their guidance on the benefits of being a TMS member as an early-career researcher in the field of materials engineering.

Along with these mentoring resources, Vijayaraghavan said that networking and collaborating on projects whenever possible are vital tools for scientists and engineers working in all sectors. "By working with a diversified group, I have been able to get valuable inputs while also concentrating on research projects which have been of high relevance to current needs and challenges."

In addition to seeking opportunities for collaboration, Vijayaraghavan left this parting thought for other scientists and engineers starting out in the field: "As a young researcher, we would be under constant pressure to publish and disseminate the research findings, as it forms the important output or measure of success. However, it is also important to focus on the relevance of work, as some universities around the world are now focusing more on the citation metrics rather than the actual count of published articles itself." 